\title{
Efficacy and Safety of Omeprazole and Amoxicillin in the Treatment of Gastric Ulcer
}

\author{
Jie Liu, Minghui Xiao, Jianping Hui*
}

Shaanxi University of Traditional Chinese Medicine, Xianyang 712000, China

\begin{abstract}
[Abstract] Objective: To study the effect of omeprazole and amoxicillin in the treatment of patients with gastric ulcer. Methods: 88 patients with gastric ulcer in our hospital from March 2018 to March 2020 were selected as research subjects and divided into experimental group (44 cases were treated with omeprazole + amoxicillin) and control group (44 cases were treated with omeprazole). The therapeutic effect, ${ }^{13} \mathrm{C}$ positive rate of breath test before and after treatment, recurrence rate within half a year and adverse reactions were compared between the two groups. Results: The total effective rate of the experimental group $(97.73 \%, 43 / 44)$ was higher than that of the control group $(84.09 \%, 37 / 44), P<0.05$; After treatment, the ${ }^{13} C$ positive rate of breath test in the experimental group $(22.73 \%, 10 / 44)$ was lower than that in the control group (47.73\%, $21 / 44), P<0.05$; The recurrence rate in the experimental group was $4.55 \%(2 / 44)$, which was significantly higher than that in the control group $(20.45 \%, 9 / 44), P<0.05$; The incidence of adverse reactions in the experimental group $(9.09 \%, 4 / 44)$ was significantly lower than that in the control group $(15.91 \%, 7 / 44), \mathrm{P}<0.05$. Conclusion: In the process of clinical treatment of gastric ulcer, omeprazole combined with amoxicillin has significant effect, not only the recurrence rate is low, but also the incidence of adverse events should be less, and the treatment is safer.
\end{abstract}

Key words: Gastric ulcer; Omeprazole; Amoxicillin; Treatment effect; Security

Publication date: May, 2021; Publication online: 31 May, 2021

*Corresponding author: Jianping Hui, 1192209646@qq.com

\section{Introduction}

The main sites of gastric ulcer mainly include gastric angle, hiatal hernia, cardia and gastric antrum. It is a kind of ulcer phenomenon, which is included in the category of digestive tract diseases. It is generally caused by irrational use of drugs, bad eating habits and Helicobacter pylori infection ${ }^{[1]}$. After the occurrence of gastric ulcer disease, the main clinical manifestations are epigastric pain, dull pain, dull pain and burning sensation, which directly affect the life and work of patients. If the condition is serious or the treatment is unreasonable, it will also cause a series of complications such as gastric perforation, which will threaten the life safety of patients. At present, the pressure of modern life and work has increased significantly, directly improving the clinical incidence rate of gastric ulcer, and the trend of younger age is significant. Drug therapy is the main clinical treatment, but the recurrence rate is high ${ }^{[2]}$. Therefore, it is necessary to deeply study and analyze the value of drug treatment for gastric ulcer patients.

\section{Material and methods}

\subsection{Baseline information}

88 patients with gastric ulcer in our hospital from March 2018 to March 2020 were selected and divided into control group and experimental group by parity method, with 44 cases in each group. There were 25 males and 19 females in the control group, with a median age of $(46.68 \pm 8.82)$ years;There were 24 males and 20 females in the experimental group, with a median age of $(46.65 \pm 8.85)$ years. Comparison of the two groups into the group, the above data showed that $\mathrm{P}>0.05$, with comparison value.

\subsection{Methods}

The control group was treated in Omeprazole capsule (H20065335, English name: Omeprazole Enteric capsule), taken twice a day, with a dose of $20 \mathrm{mg}$ each time.

The experimental group was treated with omeprazole + Amoxicillin capsule, and the usage and dosage of 
omeprazole were the same as the control group, and combined with Amoxicillin (National Drug Approval: H46020311, approval date: 2016-02-02) treatment, three times a day, each dose was $0.5 \mathrm{~g}^{[3]}$.

Two groups of patients in the drug treatment at the same time, still need to implement symptomatic treatment combined with the condition. If the patient has bleeding, it is necessary to choose proton pump inhibitors or $\mathrm{H} 2$ receptor antagonists for hemostatic treatment, so as to ensure that the $\mathrm{pH}$ value of the patient's stomach can be improved and the internal environment is more stable. If the treatment is ineffective, endoscopic treatment, surgical treatment or interventional treatment should be used. After treatment, patients are not allowed to eat indigestible food rich in dietary fiber ${ }^{[4]}$. In addition, it is not allowed to use unprocessed food with stimulating effect on gastric acid secretion. Patients should not drink alcohol or drink strong tea and coffee, follow the basic principle of eating less and eating more, try to eat 5-6 meals a day. Before eating, the food should be chopped or mashed as much as possible to relieve the gastrointestinal pressure of patients, and the temperature of the food should be moderate.

\subsection{Evaluating indicator}

(1) The treatment effect, recurrence rate in half a year and adverse reactions were observed and recorded. Among them, the evaluation standard of clinical therapeutic effect consists of three parts: markedly effective, effective and ineffective. After treatment, abdominal pain disappeared completely or basically, and gastroscopy showed that the lesion mucosa had returned to normal; Effective means that the patient's abdominal pain improved after treatment, and gastroscopy showed that the lesion mucosa improved significantly; No effect, that is, the patient's abdominal pain did not improve after treatment, and gastroscopy showed that the improvement of the lesion mucosa was not obvious. The total effective rate of clinical treatment was the sum of significant efficiency and effective rate ${ }^{[5]}$. Adverse reactions included pruritus, rash, nausea and vomiting.

(2) The ${ }^{13} C$ positive rate of breath test before and after treatment was analyzed.

\subsection{Statistical analysis}

Statistical software SPSS 17.0 processing two groups of data, $P<0.05$ means that the data is statistically significant.

\section{Results}

\subsection{Treatment effect analysis of experimental group and control group}

Data comparison between groups, $P<0.05$ ( Table 1 ).

Table 1. Comparison of treatment effect between the two groups (n /\%)

\begin{tabular}{cccccc}
\hline Group & n & Remarkable effect & Effective & Invalid & Total effective rate \\
\hline Experience group & 44 & 28 & 15 & 1 & 97.73 \\
Control group & 44 & 24 & 13 & 7 & 84.09 \\
$X 2$ & & & & 4.9500 \\
$P$ & & & & 0.0260 \\
\hline
\end{tabular}

\section{2 ${ }^{13} \mathrm{C}$ Positive rate of breath test before and after} treatment in the two groups
After treatment, the experimental group index compared with the control group, $\mathrm{P}<0.05$ (Table 2).

Table 2. Comparison of ${ }^{13} \mathrm{C}$ positive rate of breath test before and after treatment between experimental group and control group (n $/ \%)$

\begin{tabular}{cccc}
\hline Group & \multirow{n}{*}{} & \multicolumn{2}{c}{$C$ Positive rate of breath test } \\
\cline { 3 - 4 } & & Before treatment & After treatment \\
\hline Experience group & 44 & $40(90.91)$ & $10(22.73)$ \\
Control group & 44 & $38(86.36)$ & $21(47.73)$ \\
$X 2$ & & 0.4513 & 6.0260 \\
$P$ & & 0.5017 & 0.0140 \\
\hline
\end{tabular}

\subsection{Analysis of recurrence rate of experimental group and control group within half a year}


There was no significant difference between the two groups $\quad(P<0.05)$ (Table 3$)$.

Table 3. Recurrence rate in half a year (n/\%)

\begin{tabular}{ccc}
\hline Group & $\mathbf{n}$ & Recurrence rate in half a year \\
\hline Experience group & 44 & $2(4.55)$ \\
Control group & 44 & $9(20.45)$ \\
$X 2$ & & 5.0909 \\
$P$ & & 0.0240 \\
\hline
\end{tabular}

3.4 Study on adverse reactions of the two groups

that of control group $(P>0.05)$ (Table 4).

The total incidence of experimental group was higher than

Table 4. Comparison of adverse reactions between experimental group and control group (n /\%)

\begin{tabular}{cccccc}
\hline Group & n & Itch & Rash & Nausea and vomiting & Total incidence \\
\hline Experience group & 44 & 2 & 1 & 1 & $4(9.09)$ \\
Control group & 44 & 0 & 3 & 4 & $7(15.91)$ \\
$X 2$ & & & & & 0.9351 \\
$P$ & & & & 0.3335 \\
\hline
\end{tabular}

\section{Discussion}

The main pathogenesis of gastric ulcer is the decline of the protective function of human gastric mucosa, and then infected with Helicobacter pylori, or abnormal gastric acid secretion, eventually damage the mucosa and form gastric ulcer. In drug treatment, there are usually unreasonable applications, especially antipyretic and analgesic drugs, aspirin preparations and hormone replacement drugs, which can easily increase the burden of the gastrointestinal tract, bring chemical stimulation to the gastrointestinal mucosa of patients, and damage the gastric mucosa. If you eat irritant food, overeat or lack of regular diet, you will make the body's gastric mucosa subject to physical stimulation, resulting in gastric overfilling, resulting in excessive gastric acid secretion and mucosal damage ${ }^{[6]}$. In addition, long-term mental stress, smoking and drinking or poor mood are also prone to suffer from gastric ulcer. After the onset of the disease, we must pay more attention and take reasonable treatment measures to avoid adverse consequences. If gastric ulcer is not treated for a long time, it is easy to appear upper gastrointestinal bleeding, pyloric obstruction, ulcer perforation and other symptoms, and even cancer.

Therefore, the clinical treatment should focus on the inhibition of gastric acid secretion, so as to improve the gastric environment of patients and protect the gastric $\operatorname{mucosa}^{[7]}$. At the same time, we should also inhibit the reproduction of Helicobacter pylori. In the clinical research results clearly pointed out that Helicobacter pylori infection is the main cause of gastric ulcer. In order to further optimize the clinical efficacy of gastric ulcer, it is necessary to eradicate Helicobacter pylori infection and prevent the recurrence of gastric ulcer. On this basis, we should emphasize the importance of diet, try not to eat spicy and cold food, eat more light liquid food, and also reasonably supplement protein and vitamin $\mathrm{C}$, so as to ensure a balanced intake of nutrition, continuously strengthen its immune function, and give necessary protection to gastric mucus. If the patient has the performance of bleeding, it is necessary to take appropriate hemostatic treatment measures, try to improve the $\mathrm{pH}$ value in the stomach.

Omeprazole is a proton pump inhibitor, which can be easily concentrated in acidic environment. After taking omeprazole, it will be distributed in the secretory tubules of gastric parietal cells. In high acid environment, omeprazole can be transformed into sulphonamide. On the basis of combining disulfide bond and secretory membrane endoplasmic pump of parietal cells, omeprazole can regulate the internal environment of the stomach, protect the gastric mucosa, and improve the clinical manifestations of gastric ulcer. Amoxicillin belongs to semi synthetic penicillin, namely broad-spectrum antibiotics ${ }^{[8]}$. After taking amoxicillin, the drug will be hydrolyzed into peptide bonds, which will bind to the transpeptidase in the bacteria in a 
short time, making its activity lost. Transpeptidase plays an important role in the synthesis of glycopeptides and the construction of cell wall. After inhibiting the activity of transpeptidase, the bacteria will be affected by the cell wall defect and continuously permeate water, which makes the bacteria form globules in a short time, and then dissolve, rupture and die. According to the existing research results, amoxicillin combined with omeprazole can kill Helicobacter pylori in stomach and duodenum, improve the clinical performance of patients with peptic ulcer, and effectively reduce the recurrence rate of ulcer.

In the study, the experimental group was treated with amoxicillin combined with omeprazole, compared with the control group (except for adverse reactions), $P<0.05$. The results showed that after taking omeprazole and amoxicillin, the treatment effect of gastric ulcer patients was much better than that of the control group, and the ${ }^{13} C$ positive rate of breath test was lower than that of the control group, and the recurrence rate within half a year was low, which could be used to cure Helicobacter pylori. The main cause of recurrence of gastric ulcer disease is Helicobacter pylori, which can reduce the recurrence rate after treatment. The adverse reactions of the two drugs are not serious, so the treatment safety is high.

In general, omeprazole combined with amoxicillin in the clinical treatment of gastric ulcer patients, the effect is ideal, especially the treatment effect of Helicobacter pylori is more obvious, which can reduce the recurrence rate of patients after treatment. At the same time, the combination of the two drugs will not appear too many adverse reactions, so the treatment is safer and has high clinical promotion value.

\section{References}

[1] Chen J. Effect of amoxicillin combined with omeprazole on gastrointestinal function of elderly patients with gastric ulcer [J]. World's Latest Medical Information Abstracts (continuous electronic journal), 2020,20 (46): 117-118.

[2] Ni Y. Efficacy of omeprazole combined with amoxicillin in the treatment of gastric ulcer and its effect on oxidative stress level of patients $[\mathrm{J}]$. Chinese Journal of Modern Drug Application, 2020,14 (20): 7-10.

[3] Yang N. Efficacy and safety of omeprazole combined with amoxicillin in the treatment of gastric ulcer $[\mathrm{J}]$. Jiayou Yunbao, 2020, 2 (4): 119-120.

[4] Cheng SY. Application study and safety analysis of omeprazole combined with amoxicillin in patients with gastric ulcer [J]. China Health Nutrition, 2019,29 (35): 311-312.

[5] Mu HJ. To observe the clinical effect of omeprazole combined with amoxicillin in the treatment of gastritis and gastric ulcer [J]. Healthy Vision, 2019 (5): 75-76.

[6] Liao CL. Clinical effect and adverse reaction rate of omeprazole combined with amoxicillin in the treatment of gastritis and gastric ulcer [J]. Journal of General Stomatology (Electronic Edition), 2019, 6(3): 158-159.

[7] Xie ZH. Clinical efficacy and safety evaluation of omeprazole, amoxicillin and furazolidone triple therapy on gastric ulcer patients with Helicobacter pylori infection [J]. Family Medicine, 2019 (10): 195-196.

[8] Ren WM. Application value of omeprazole combined with amoxicillin and metronidazole and combined with comprehensive nursing in patients with gastric ulcer $[\mathrm{J}]$. Friends of Health, 2019 (20): 3 